\title{
Multifunctional Materials for Emerging Technologies
}

\author{
Dr. F. Rosei \\ Institut National de la Recherche Scientifique \\ Varennes (QC), Canada \\ rosei@emt.inrs.ca
}

\begin{abstract}
As the age of fossil fuels is coming to an end, now more than ever there is the need for more efficient and sustainable renewable energy technologies. This presentation will give an overview on recent developments in solar technologies that aim to address the energy challenge. In particular, nanostructured materials synthesized via the bottom-up approach present an opportunity for future generation low cost manufacturing of devices [1]. We demonstrate various multifunctional materials, namely materials that exhibit more than one functionality, and structure/property relationships in such systems, including new strategies for the synthesis of multifunctional nanoscale materials to be used for applications in photovoltaics, solar hydrogen production, luminescent solar concentrators and other emerging optoelectronic technologies. [2-31].
\end{abstract}

\section{References}

[1] F. Rosei, J. Phys. Cond. Matt. 16, S1373 (2004); [2] C. Yan et al., Adv. Mater. 22, 1741 (2010); [3] C. Yan et al., J. Am. Chem. Soc. 132, 8868 (2010); [4] R. Nechache et al., Adv. Mater. 23, 1724 (2011); [5] R. Nechache et al., Appl. Phys. Lett. 98, 202902 (2011); [6] G. Chen et al., Chem. Comm. 48, 8009 (2012); [7] G. Chen et al., Adv. Func. Mater. 22 , 3914 (2012); [8] R. Nechache et al., Nanoscale 4, 5588 (2012); [9] J. Toster et al., Nanoscale 5, 873 (2013); [10] T. Dembele et al., J. Power Sources 233, 93 (2013); [11] S. Li et al., Chem. Comm. 49, 5856 (2013); [12] T. Dembele et al., J. Phys. Chem. C 117, 14510 (2013); [13] R. Nechache et al., Nature Photonics 9, 61 (2015); [14] R. Nechache et al., Nanoscale 8, 3237 (2016); [15] R. Adhikari et al. Nano Energy 27, 265 (2016); [16] H. Zhao et al., Small 12, 3888 (2016); [17] J. Chakrabartty et al., Nanotechnology 27, 215402 (2016); [18] D. Benetti et al., J. Mater. Chem. C 4, 3555 (2016); [19] K. Basu et al., Sci. Rep. 6, 23312 (2016); [20] Y. Zhou et al., Adv. En. Mater. 6, 1501913 (2016); [21] H. Zhao et al., Nanoscale 8, 4217 (2016); [22] L. Jin et al., Adv. Sci. 3, 1500345 (2016); [23] H. Zhao et al., Small 11, 5741 (2015); [24] S. Li et al., Small 11, 4018 (2015); [25] K.T. Dembele et al., J. Mater. Chem. A 3, 2580 (2015); [26] H. Zhao et al., Nano Energy 34, 214-223 (2017); [27] S. Li et al., Nano Energy 35, 92-100 (2017); [28] G.S. Selopal et al., Adv. Func. Mater. 27, 1401468 (2017); [29] X. Tong et al., Adv. En. Mater. 8, 1701432 (2018); [30] H. Zhao, F. Rosei, Chem 3, 229-258 (2017); [31] J. Chakrabartty et al., Nature Phot. 12, 271-276 (2018). 